\title{
Effect of Repetitive Transcranial Magnetic Stimulation on Meta-Worry and Neuropsychological Functions among Patients with Depression
}

\author{
Mahboobeh Majdi1 ${ }^{1}$, Maryam Bakhtiyari2 ${ }^{2}$, , Reza Rostami ${ }^{3}$, Abbas Masjedi Arani ${ }^{4}$ and Mohsen Saberi ${ }^{5}$ \\ 1 Department of Clinical Psychology, School of Medicine, Shahid Beheshti University of Medical Sciences, Tehran, Iran \\ $2 \mathrm{PhD}$, Associate Professor, Department of Clinical Psychology, Faculty of Medicine, Shahid Beheshti University, Tehran, Iran \\ 3 PhD, Professor, Department of Psychology, Faculty of Educational Sciences and Psychology, Tehran University, Tehran, Iran \\ ${ }^{4} \mathrm{PhD}$, Associate Professor, Department of Psychology, Faculty of Medicine, Shahid Beheshti University, Tehran, Iran \\ 5 Associate Professor of Community Medicine, Quran and Hadith Research Center and Department of Community Medicine, Faculty of Medicine, Tehran, Iran
}

* Corresponding author: Maryam Bakhtiyari, Department of Clinical Psychology, Faculty of Medicine, Shahid Beheshti University, Tehran, Iran. Email: hosseini.somay@gmail.com

Received 2020 November 26; Revised 2020 December 28; Accepted 2021 January 13.

\begin{abstract}
Background: Repetitive transcranial magnetic stimulation (rTMS) is recommended as an effective treatment for both major depressive disorder (MDD) and treatment-resistant depression.

Objectives: According to the possible impact of rTMS on cognitive psychological characteristics, this study aimed to determine the effectiveness of rTMS in meta-worry and neuropsychological functions among MDD patients.

Methods: This quasi-experimental study was conducted on 30 patients with MDD referring to Atieh Clinical Neuroscience Center, Tehran, Iran, in 2019. The participants were randomly divided into two groups of intervention and control $(\mathrm{n}=15$ each). The data collection tools included the Beck Depression Inventory, meta-worry subscale of the Anxious Thoughts Inventory, and Cambridge Neuropsychological Test Automated Battery.

Results: The comparison between the two groups showed that the scores of the meta-worry scale improved after the intervention in patients undergoing rTMS, compared to those in the control group $(\mathrm{Z}=-3.41 ; \mathrm{P}=0.002)$; however, no difference was observed between the two groups in the follow-up $(\mathrm{Z}=-2.02 ; \mathrm{P}=0.053)$. The assessment of neuropsychological functions among the patients undergoing rTMS and those in the control demonstrated that neuropsychological functions (i.e., Minimum Spanning Tree, Rapid Visual Information Processing, and Spatial Working Memory) were significantly different immediately after the intervention and in the follow-up ( $\mathrm{P}<0.05)$ except for the Difficulty Maintaining Sleep (DMS) subtest. In addition, the mean depression score was significantly different between the two groups $(\mathrm{Z}=-4.17 ; \mathrm{P}<0.005)$. There was a significant relationship between depression and all the subtests of neuropsychological functions except for DMS $(\mathrm{P}>0.05)$.

Conclusion: In summary, the results of the current study indicated that the use of rTMS was an effective method in the improvement of neuropsychological functions except for DMS in patients with depression. However, the obtained findings did not demonstrate the persistent effect of multiple rTMS on meta-worry.
\end{abstract}

Keywords: Depression, Meta-worry, Neuropsychological functions, Transcranial magnetic stimulation

\section{Background}

Depression is the most common mental disorder after anxiety and is associated with several symptoms, including a lack of motivation, loss of appetite, decreased social functioning, sleep disorders, and isolation (1). The prevalence rate of depression is reported within the range of $5-17 \%$ around the world (2) and estimated at 25\% in Iran (3). Currently, due to the abundance of etiological factors, such as social status, abnormal lifestyle, economic status, environmental stress, and chronic diseases, this disorder has become highly prevalent (4).

One of the issues that patients suffering from depression deal with is meta-worry. The beliefs and negative evaluations of anxiety, such as worrying about anxiety, are called meta-worry. Meta-worry involves the beliefs about the positive and negative effects of worry. It plays the main role in the development and maintenance of generalized anxiety disorder (5). Another problem of patients with depression is related to their neuropsychological functions (6). Working memory is highly important for many higher-level cognitive functions, such as problem-solving, reasoning, planning, and behavior guiding. These functions are affected in patients suffering from depression with problems of neuropsychological functions (7).

Antidepressants are the first-line treatments adopted for mood disorders, especially depression. In addition, electroconvulsive therapy (ECT) is performed only for patients who do not respond to medication or are intolerant to medication (8). However, response to antidepressants is variable; accordingly, fewer than half of depressed patients respond to their first drug treatment, leading to the prolongation of treatment time and an increase in medical costs (9). Psychotherapy and ECT are suggested as viable options for the treatment of nonresponders with major depressive disorder (MDD) (10). However, the application of the aforementioned approaches is involved with several problems, such 
as treatment discontinuation due to the long duration of psychotherapeutic treatments and possible risks of ECT (11), which are performed only for patients who do not respond to medication or are intolerant to medication (12).

In general, more than a third of patients with treatment-resistant depression (TRD) continue to suffer from residual symptoms $(13,14)$. Treatmentresistant depression is a multiple risk factor and complex clinical problem, which should be targeted by integrated therapeutic strategies (e.g., the optimization of medications and psychosocial and cultural therapies) and somatic therapies (e.g., ECT, repetitive transcranial magnetic stimulation [rTMS], and deep brain stimulation) (13). In this regard, rTMS is recommended by the Food and Drug Administration for the treatment of both MDD and TRD. Transcranial magnetic stimulation acts on the brain areas involved in the pathogenesis of MDD (15). It is a non-invasive and safe procedure affecting the cortical activity of the stimulated area by sending electric currents that modify the glucose levels and activity of neurotransmitters in that area (16).

The results of some studies showed that rTMS is effective in the treatment of triple signs and symptoms of depression (i.e., cognition, body, and negativeworthlessness). The rTMS affected the left posterior cortex, which improved the neuropsychological function of patients with depression (17). Pirmoradi et al. observed that multiple TMS reduced cognitive and physical symptoms, pessimism, and feelings of worthlessness in patients with depression (18). According to another study performed by Durmaz et al., multiple rTMS decreased the symptoms of depression and anxiety (19). The effectiveness of multiple magnetic stimulations of the brain in working memory and depression is also confirmed by some studies $(20,21)$.

Although the effectiveness of rTMS in the cognitive function of patients with depression is widely evaluated, a limited number of studies assessed the effect of rTMS on meta-worry, as the main issue patients suffering from depression are dealing with. Meta-worry is a part of the metacognitive model which has been studied in patients with obsessive-compulsive disorder (22). It seems that there is a relationship between metaworry and neurocognitive abilities in patients suffering from depression. The findings of functional neuroimaging studies showed a decrease in the activity of the left prefrontal cortex, especially in Brodmann areas (i.e., BA9 and BA46), in depressed patients. Moreover, pieces of evidence have been indicative of the activation changes in the corticosubcortical network (i.e., subgenual and anterior cingulate cortices) among the patients (23). However, a heterogeneous neuropsychological performance was reported among depressed patients; therefore, it is necessary to consider the differences between patients in terms of cognitive deficits (24).

The investigation of treatment methods in patients with financial depression is of great importance due to the high prevalence of depression, cognitive psychological problems of the patients, and impact of depression on the quality of life.

\section{Objectives}

According to the possible effect of rTMS on the cognitive psychological characteristics of patients and inadequacy of performed studies on the effects of the above-mentioned treatments on meta-worry and neuropsychological functions, this study was conducted to determine the effectiveness of rTMS in meta-worry and neuropsychological functions among patients with depression.

\section{Methods}

This quasi-experimental study was conducted on a total of 30 patients with MDD referring to Atieh Clinical Neuroscience Center in Tehran, Iran, in 2019.

\subsection{Inclusion and Exclusion Criteria}

Patients with a definite diagnosis of MDD (score of $\geq 20$ based on the Beck Depression Inventory), the age range of 20-50 years, and junior high school education or higher were entered into this study. Moreover, the eligible patients had no history of mindfulness-based cognitive therapy or stressful events, such as death and divorce in the past 3 months. The exclusion criteria were pregnancy, history of concussion or seizures in the family, bipolar disorder, psychotic disorders, psychotropic or substance abuse, and metal implant/prosthesis or heart rate induction device. In addition, patients with a comorbid neurological or psychiatric diagnosis were excluded from the study. The absence of more than one session and incomplete questionnaires were also other exclusion criteria.

\subsection{Research tools}

\subsubsection{Beck Depression Inventory}

This 21-item questionnaire is scored on a 4-point Likert scale (0-3). The total score of the questionnaire is calculated based on the sum of the scores of the items, rendering for the range of 0-63, with higher scores indicating higher levels of depression. In this tool, the score ranges of $0-10,11-16,17-20,21-30,31-$ 40 , and $>40$ are respectively considered normal, slight, mild, moderate, severe, and very severe depression. The predictive validity of this instrument was confirmed in Iran, and its reliability was estimated at 0.89 by Cronbach's alpha coefficient (18).

\subsubsection{Meta-worry subscale}

This 7-item questionnaire is used for the assessment of anxiety level and is scored on a 4-point 
Likert scale (1-4). The total score of the questionnaire is calculated by adding the scores of the items. The scores are within the range of 7-28 with higher scores indicating higher levels of meta-worry. The validity of this tool was confirmed, and its reliability was reported to be 0.75 using Cronbach's alpha coefficient (25).

\subsubsection{Neuropsychological Function Test}

This 7-item questionnaire measures worry and metacognition through two scales. One scale assesses the frequency of meta-worry on a 4-point Likert scale $(1=$ Never, $2=$ Sometimes, $3=0 \mathrm{ften}$, and $4=$ Almost always). The other scale is used to rate the belief in each meta-worry at the time of occurrence with the total range score of $0-100$. This test has four major categories, including attention and psychomotor abilities, executive functions, memory, and emotional and social cognition. To evaluate the neuropsychological functions of the participants, four tools were used, namely Motor Planning Task (MOT), Rapid Visual Information Processing (RVP), Spatial Working Memory (SWM), and Difficulty Maintaining Sleep (DMS) subscales of the Cambridge Neuropsychological Test Automated Battery. The MOT subtest measures the overall level of motor impairment or lack of comprehension. The RVP measures sustained attention, and the SWM evaluates the ability to retrieve spatial information and manipulate them in working memory. Furthermore, the DMS assesses the ability to remember abstract and complex visual patterns and is sensitive to the function of the medial temporal lobe (26).

\subsection{Study Design}

The sample size of this study $(n=30)$ was estimated based on a study carried out by Sharifi Saki et al. (27) using the following equation:

$$
\mathrm{n}=\frac{2 \sigma^{2}\left(\mathrm{z}_{\left.1-\frac{\alpha}{2}+\mathrm{z}_{1-\beta}\right)^{2}}\right.}{\mathrm{d}^{2}}=\frac{2(1 / 67)^{2}(1 / 96+1 / 28)^{2}}{4 / 507}=12 / 991
$$

In total, eligible subjects were selected using the convenience sampling method with a power of 0.9 and $\alpha$ of 0.05 , which was calculated at 12.9 for each group. However, 15 participants were entered in each group to increase the power of the test. Firstly, the subjects were assigned a code and then randomly divided into two groups of intervention $(n=15)$ and control $(\mathrm{n}=15)$. The patients and examiner were blinded to the allocation status.

The participants in the intervention and control groups were previously treated with concomitant antidepressants. The patients in the intervention group received multiple rTMS, and those in the control group were put on a waiting list for training. For the intervention, Neurosoft Transcranial Magnetic Stimulator (Neurosoft, Ivanovo, Russia)was used to stimulate the dorsolateral prefrontal cortex by creating magnetic fields (i.e., bilaterally over the dorsolateral prefrontal cortex). Electric currents, after passing through the coil that is placed on the head of the patient, generate magnetic fields leading to a lighter electric current in the cerebral cortex which stimulates the target nerve tissue. Each of the two stimuli had a frequency and intensity of $10 \mathrm{~Hz}$ and $120 \%$ motor threshold, respectively, and lasted for $5 \mathrm{sec}$ with a 10 -second interval. This mechanism was performed on the posterior cortex of the left forehead for 5 weeks and four 30-minute sessions each week. The rTMS on the dorsolateral prefrontal cortex was performed by a specialist in Atieh Clinical Neuroscience Center, Tehran, Iran.

The required data were collected using a demographic characteristic form, including gender, age, and educational level. Moreover, the Beck Depression Inventory, a meta-worry subscale of Anxious Thoughts Inventory, and Neuropsychological Function Test were employed as other tools to gather the required data. The patients were assessed in terms of depression, meta-worry, and neuropsychological functions before the intervention and immediately and 3 months after the intervention.

\subsection{Statistical Analysis}

The collected data were analyzed in SPSS software (version 23) using the Shapiro-Wilk test (to assess the normality of the data), Levene's test (to measure the homogeneity of variance and covariance), and Mauchly's test of sphericity (to calculate the homogeneity of covariance). Independent t-tests, paired t-test, and analysis of variance were also utilized for quantitative variables. Additionally, the Chi-square test was employed for qualitative variables. A p-value of less than 0.05 was considered significant.

\subsection{Ethical considerations}

This article was derived from a thesis submitted for the partial fulfillment of the requirement for a $\mathrm{PhD}$ in clinical psychology to Shahid Beheshti University of Medical Sciences, Tehran, Iran, and Health Services in Tehran. The protocol of the present study was approved by the Research Ethics Committee of Shahid Beheshti University of Medical Sciences. This study was conducted according to the Helsinki Human Rights criteria (ethics code of IR.SBMU.MSP.REC.1397.95). Before the initiation of the study, the objectives were explained to the patients, and informed consent was obtained from all the study participants. The patients were assured that their information would remain confidential and they could withdraw from the study at any time.

\section{Results}

A total of 30 patients were selected and divided into the intervention and control groups. It should be 


\begin{tabular}{|c|c|c|c|c|c|c|c|c|c|}
\hline \multirow[b]{2}{*}{ Variable } & \multirow[b]{2}{*}{ Category } & \multicolumn{2}{|c|}{ Intervention } & \multicolumn{2}{|c|}{ Control } & \multicolumn{2}{|c|}{ Total } & \multirow[b]{2}{*}{$\chi^{2}$} & \multirow[b]{2}{*}{ P-value } \\
\hline & & $\mathbf{n}$ & $\%$ & $\mathbf{n}$ & $\%$ & $\mathbf{n}$ & $\%$ & & \\
\hline \multirow{2}{*}{ Gender } & Male & 5 & 33.3 & 5 & 33.3 & 10 & 33.3 & \multirow{2}{*}{0.61} & \multirow{2}{*}{0.73} \\
\hline & Female & 10 & 66.7 & 10 & 66.7 & 20 & 66.7 & & \\
\hline \multirow{3}{*}{ Age (year) } & $21-30$ & 3 & 20.0 & 2 & 13.3 & 5 & 16.7 & \multirow{3}{*}{0} & \multirow{3}{*}{1} \\
\hline & $31-40$ & 5 & 33.3 & 7 & 46.7 & 12 & 40.0 & & \\
\hline & $41-50$ & 7 & 46.7 & 6 & 40.0 & 13 & 43.3 & & \\
\hline \multirow{4}{*}{$\begin{array}{l}\text { Educational } \\
\text { level }\end{array}$} & Junior high school & 1 & 6.67 & 2 & 13.33 & 3 & 10 & \multirow{4}{*}{0.72} & \multirow{4}{*}{0.86} \\
\hline & High school & 3 & 20 & 2 & 13.33 & 5 & 16.7 & & \\
\hline & Associate degree & 5 & 33.3 & 4 & 26.67 & 9 & 30 & & \\
\hline & Bachelor's degree & 6 & 40 & 7 & 46.67 & 11 & 36.7 & & \\
\hline
\end{tabular}

mentioned that there was no sample attrition. The age mean values of the subjects in the intervention and control groups were obtained at $37.6 \pm 95.29$ and $37.11 \pm 6.48$ years, respectively. Table 1 tabulates the comparison of demographic characteristics, indicating no significant difference between the studied groups in terms of gender, age, and educational level $(P>0.05)$. In addition, the mean scores of depression before and after the intervention were estimated at 29.3 and 19.4, respectively. The comparison of depression scores after the intervention showed a significant difference between the two groups $(\mathrm{Z}=-4.17 ; \mathrm{P}<0.005)$.

Table 2 summarizes the comparison of metaworry and neuropsychological functions of the subjects in the intervention and control groups. The obtained results showed that there was no significant difference in the meta-worry and neuropsychological function subtests between the two groups at the baseline, indicating that two groups were matched in terms of these variables. The comparison between the two groups demonstrated that the scores of the meta-worry scale improved after the intervention in patients undergoing rTMS, in comparison to those in the control group $(\mathrm{Z}=-3.41 ; \mathrm{P}=0.002)$; however, no difference was observed between the two groups in the follow-up $(\mathrm{Z}=-2.02 ; \mathrm{P}=0.053)$. Moreover, the assessment of neuropsychological functions among the patients undergoing rTMS and those in the control group showed that the scores of MST, RVP, and SWM were significantly different immediately after the intervention and in the follow-up $(\mathrm{P}<0.05)$; nevertheless, no difference was observed in DMS scores immediately after the intervention and in the follow-up between the two groups $(\mathrm{P}>0.05)$

Table 3 presents the effect of rTMS on meta-worry and neuropsychological functions, showing that the effect of group and time-group interaction was significant on all variables except for DMS $(\mathrm{P}<0.05)$. Therefore, it can be said that the difference between the mean score of variables in the different stages of the study was significant. Table 4 tabulates the comparison of the baseline stage with the posttreatment and follow-up stages based on the Bonferroni test. Regarding, the mean value of the baseline stage had a significant difference with those reported for the post-treatment and followup stages regarding the meta-worry and neuropsychological function variables $(\mathrm{P}<0.05)$. However, the difference between the mean values of the post-treatment and follow-up stages was not significant for any of the variables $(P>0.05)$. In other words, multiple TMS significantly reduced meta-worry and improved neuropsychological functions in patients with depression in the posttreatment and follow-up stages, compared to those reported for the baseline stage.

\begin{tabular}{|c|c|c|c|c|c|c|c|}
\hline \multirow{2}{*}{ Variable } & \multirow[t]{2}{*}{ Stage } & \multicolumn{2}{|c|}{ Intervention group } & \multicolumn{2}{|c|}{ Control group } & \multirow[t]{2}{*}{ Statistical test } & \multirow[t]{2}{*}{ P-value } \\
\hline & & Mean & Standard deviation & Mean & Standard deviation & & \\
\hline \multirow{3}{*}{ Meta-worry } & Baseline & 11 & 5.34 & 13.13 & 4.22 & $0.84^{*}$ & 0.25 \\
\hline & Post-treatment & 6.86 & 3.97 & 12.13 & 4.45 & -3.41 & 0.002 \\
\hline & Follow-up & 8.66 & 3.9 & 11.6 & 4.03 & -2.02 & 0.053 \\
\hline \multirow{3}{*}{$\begin{array}{l}\text { Motor } \\
\text { Screening Task }\end{array}$} & Baseline & 50.93 & 5.18 & 49.8 & 3.52 & $-0.02^{*}$ & 0.98 \\
\hline & Post-treatment & 65.73 & 10.13 & 52.6 & 5.52 & $-3.66^{*}$ & $<0.005$ \\
\hline & Follow-up & 62.86 & 8.95 & 53.46 & 4.68 & $-3.12^{*}$ & 0.002 \\
\hline \multirow{3}{*}{$\begin{array}{l}\text { Rapid Visual } \\
\text { Information } \\
\text { Processing }\end{array}$} & Baseline & 49.46 & 4.307 & 51.2 & 3.72 & -1.1 & 0.24 \\
\hline & Post-treatment & 56.73 & 3.88 & 51 & 3.52 & 4.23 & $<0.005$ \\
\hline & Follow-up & 56.06 & 3.88 & 50.66 & 3.71 & 3.8 & 0.001 \\
\hline \multirow{3}{*}{$\begin{array}{l}\text { Spatial } \\
\text { Working } \\
\text { Memory }\end{array}$} & Baseline & 51 & 5.73 & 50.8 & 6.81 & 27.2 & 0.93 \\
\hline & Post-treatment & 58.6 & 6.46 & 50.73 & 7.15 & $-3.27^{*}$ & 0.001 \\
\hline & Follow-up & 57.33 & 9.54 & 46.26 & 5.13 & $-3.14^{*}$ & 0.001 \\
\hline \multirow{3}{*}{$\begin{array}{l}\text { Delayed } \\
\text { Matching to } \\
\text { Sample }\end{array}$} & Baseline & 50.4 & 5.56 & 49.93 & 6.54 & 0.21 & 0.83 \\
\hline & Post-treatment & 56.2 & 9.19 & 50.26 & 8.26 & 1.85 & 0.07 \\
\hline & Follow-up & 55.66 & 7.84 & 50.73 & 8.87 & 1.61 & 0.11 \\
\hline
\end{tabular}


Majdi M et al.

\begin{tabular}{|c|c|c|c|c|c|c|c|}
\hline Variable & Source of effect & Sum square & Degrees of freedom & Mean square & F-statistic & P-value & Effect size \\
\hline & Group & 146.23 & 1 & 146.23 & 3.55 & 0.02 & 0.14 \\
\hline \multirow[t]{2}{*}{ Meta-worry } & Time & 179.30 & 1.21 & 147.72 & 11.99 & 0.001 & 0.22 \\
\hline & Interaction & 41.10 & 2.43 & 16.93 & 1.37 & 0.042 & 0.06 \\
\hline Motor & Group & 717.56 & 1 & 717.56 & 8.13 & 0.001 & 0.27 \\
\hline Screening & Time & 2053.53 & 1.54 & 1334.77 & 48.95 & 0.001 & 0.54 \\
\hline Task & Interaction & 567.67 & 3.08 & 184.49 & 6.77 & 0.001 & 0.24 \\
\hline Rapid Visual & Group & 130.09 & 1 & 130.09 & 4.93 & 0.012 & 0.19 \\
\hline Information & Time & 409.66 & 1.74 & 235.86 & 25.74 & 0.001 & 0.38 \\
\hline Processing & Interaction & 2790.10 & 3.47 & 80.34 & 8.77 & 0.001 & 0.3 \\
\hline Spatial & Group & 472.94 & 1 & 472.94 & 3.72 & 0.032 & 0.15 \\
\hline Working & Time & 441.08 & 1.50 & 294.64 & 12.40 & 0.001 & 0.23 \\
\hline Memory & Interaction & 524.96 & 2.99 & 175.34 & 7.38 & 0.001 & 0.26 \\
\hline Delayed & Group & 161.62 & 1 & 161.62 & 1.43 & 0.251 & 0.06 \\
\hline Matching to & Time & 276.40 & 1.97 & 140.22 & 3.96 & 0.02 & 0.09 \\
\hline Sample & Interaction & 138.49 & 3.94 & 35.13 & 0.99 & 0.43 & 0.05 \\
\hline
\end{tabular}

No significant difference was observed between the post-treatment and follow-up stages in terms of any of the variables. The significant difference between the baseline and post-treatment stages indicated the effect of the intervention. Moreover, the significant difference between the baseline and follow-up stages suggested the maintenance of the influence of the intervention on the follow-up phase. Table 5 presents the correlation between depression and neuropsychological functions. Based on the obtained results, there was a significant relationship between depression and all the subtests of neuropsychological functions after the intervention except for DMS $(\mathrm{P}>0.05)$.

\begin{tabular}{|c|c|c|c|c|c|}
\hline \multirow[t]{2}{*}{ Variable } & \multicolumn{2}{|c|}{ Evaluation stage } & \multirow{2}{*}{$\begin{array}{c}\text { Mean difference } \\
2.73^{*}\end{array}$} & \multirow{2}{*}{$\begin{array}{c}\text { Standard deviation } \\
0.67\end{array}$} & \multirow{2}{*}{$\frac{\text { P-value }}{0.001}$} \\
\hline & Baseline & Post-treatment & & & \\
\hline \multirow[t]{2}{*}{ Meta-worry } & Baseline & Follow-up & $1.98^{*}$ & 0.69 & 0.021 \\
\hline & Post-treatment & Follow-up & -0.76 & 0.56 & 0.09 \\
\hline \multirow{3}{*}{$\begin{array}{l}\text { Motor Screening } \\
\text { Task }\end{array}$} & Baseline & Post-treatment & $-8.64 *$ & 1.10 & 0.001 \\
\hline & Baseline & Follow-up & $-7.84^{*}$ & 1.08 & 0.001 \\
\hline & Post-treatment & Follow-up & 0.80 & 0.65 & 0.67 \\
\hline \multirow{3}{*}{$\begin{array}{l}\text { Rapid Visual } \\
\text { Information } \\
\text { Processing }\end{array}$} & Baseline & Post-treatment & $-4.03 *$ & 0.59 & 0.001 \\
\hline & Baseline & Follow-up & $-3.20 *$ & 0.69 & 0.001 \\
\hline & Post-treatment & Follow-up & 0.84 & 0.48 & 0.26 \\
\hline \multirow{3}{*}{$\begin{array}{l}\text { Spatial Working } \\
\text { Memory }\end{array}$} & Baseline & Post-treatment & $-4.42^{*}$ & 0.98 & 0.001 \\
\hline & Baseline & Follow-up & $-2.40^{*}$ & 0.58 & 0.001 \\
\hline & Post-treatment & Follow-up & 2.02 & 1.03 & 0.17 \\
\hline \multirow{3}{*}{$\begin{array}{l}\text { Delayed Matching } \\
\text { to Sample }\end{array}$} & Baseline & Post-treatment & $-3.27^{*}$ & 1.28 & 0.04 \\
\hline & Baseline & Follow-up & $-2 / 73^{*}$ & 1.17 & 0.04 \\
\hline & Post-treatment & Follow-up & 0.53 & 1.28 & 0.99 \\
\hline
\end{tabular}

\begin{tabular}{llcc}
\hline \multicolumn{2}{l}{ Table 5. Correlation between depression with meta-worry and neuropsychological functions } \\
\cline { 3 - 3 } Variable & \multirow{2}{*}{ Time } & \multicolumn{2}{c}{ Depression } \\
\cline { 2 - 3 } Meta-worry & Post-treatment & 0.67 & P-value \\
\hline \multirow{2}{*}{ Motor Screening Task } & Follow-up & 0.52 & 0.005 \\
\multirow{2}{*}{ Rapid Visual Information Processing } & Post-treatment & -0.52 & 0.003 \\
& Follow-up & -0.42 & 0.003 \\
\multirow{2}{*}{ Spatial Working Memory } & Follow-treatment & -0.29 & 0.11 \\
& Post-treatment & -0.47 & 0.008 \\
Delayed Matching to Sample & Follow-up & -0.35 & 0.22 \\
& Post-treatment & -0.35 & 0.052 \\
\hline
\end{tabular}

\section{Discussion}

In summary, considering the results of correlation analysis between possible changes in depression scores and changes in meta-worry and cognitive measurements, the improvements in neuropsychological functioning (and meta-worry) were associated with the improvements in depression. The scores of the meta-worry scale improved after the intervention in the patients undergoing rTMS than those in the control group; however, the obtained data did not confirm the persistent effect of rTMS on meta-worry in MDD patients. Additionally, rTMS enhanced neuropsychological functions (i.e., MST, 
RVP, and SWM) except for DMS.

It was revealed that the meta-worry scores decreased in the post-treatment and follow-up stages, compared to those reported for the baseline stage; however, there was no difference between the metaworry scores obtained in the post-test and follow-up stages. The aforementioned results are consistent with the findings of the previous studies in this regard $(17-19,28)$. Similarly, the results of a study performed by Cirillo et al. showed that TMS could reduce meta-worry (25). Durmaz et al. reported that multiple rTMS decreased anxiety symptoms (19).

It is suggested that multiple rTMS be considered a new technique for the treatment of depression. In this method, the magnetic field allows the depolarization of cortical nerve cells by sending pulses to the skull. In addition, different from electrical stimulation, TMS allows focal brain stimulation in any area and does not require anesthesia or lead to complications, such as long-term cognitive issues. Since this method stimulates the brain, it has long-term effects that can reduce meta-worry in patients with depression, which is indicative of the positive effects of this treatment $(29,30)$.

According to the results of the present study, multiple rTMS improved the neuropsychological functions of patients with depression in the posttreatment and follow-up stages. However, no significant difference was observed between the subjects' neuropsychological functions in the posttreatment and follow-up stages, which is consistent with the results of previous studies $(27,31)$. The findings of a study conducted by Rahimi et al. revealed the effect of multiple TMS of the left posterior cortex on the improvement of neuropsychological functions in patients with depression (17). Taherifard et al. demonstrated that transcranial direct-current stimulation enhanced cognitive functions, such as short-term memory, visual-spatial skills, executive functions, attention, concentration, working memory, language, and awareness of time and space (28).

In another study, Vanderhasselt et al. showed that multiple TMS improved the working memory of patients with depression (21). Based on the findings of a study carried out by Asbaghi et al., the reduction of meta-worry by multiple TMS could be attributed to its long-term potentiation mechanism and a strong continuous synaptic transmission resulting from this strong synaptic activity. This mechanism is a widely accepted model of neural flexibility as the underlying hypothesis of learning and memory. Non-invasive cortical stimulation, along with memory improvement, facilitates the increase in the effects of the long-term potentiation mechanism (27).

Many psychological disorders in patients with mood disorders improve after a variety of therapeutic interventions. However, it should be noticed that multiple TMS independently enhances various aspects of neuropsychological functions. This stimulation affected neuropsychological functions due to the high number of pulses used in the current study, compared to those in the previous studies. The intensity of stimulation can also be an effective factor. Furthermore, when the mean age of the patient increases, high-intensity stimulation is usually used to compensate for cerebral atrophy.

\subsection{Limitations and Recommendations}

The most important limitations of this study included the use of the convenience sampling method, relatively small sample size, lack of comparison of the results regarding the gender, and use of self-reporting tools for the diagnosis of depression. Another limitation was related to the lack of any other intervention method to compare its effects with those of the multiple TMS. Consequently, it is recommended to carry out further studies to use the random sampling method, consider a larger sample size for each group, compare the results based on gender, and use semi-structured interviews for the assessment of depression. Furthermore, the effect of multiple TMS on other cognitive psychological variables, such as rumination, should also be investigated and compared to those reported for other treatment methods, including neurofeedback. In addition, it is suggested that therapists use multiple TMS for health-related interventions, along with other treatments, especially for the reduction of meta-worry and improvement of neuropsychological functions in patients with depression.

\section{Conclusion}

In conclusion, the results of the present study indicated that the use of multiple rTMS was effective for the improvement of neuropsychological functions except for the DMS in patients with depression. However, the obtained findings did not demonstrate the persistent effect of multiple rTMS on meta-worry. Furthermore, the results confirmed the relationship between depression with metaworry and neuropsychological functions.

\section{Acknowledgments}

The authors would like to express their sincere gratitude to the officials of Atieh Clinical Neuroscience Center, especially Dr. Reza Rostami, and the participants who contributed to performing this research.

\section{Footnotes}

Conflict of Interests: The authors declare that there is no conflict of interest.

Funding/Support: The current research project was funded by Shahid Beheshti University of Medical Sciences. 


\section{References}

1. World Health Organization. Depression and other common mental disorders: global health estimates. Geneva: World Health Organization; 2017.

2. Lim GY, Tam WW, Lu Y, Ho CS, Zhang MW, Ho RC. Prevalence of depression in the community from 30 countries between 1994 and 2014. Sci Rep. 2018;8(1):2861. doi: 10.1038/s41598-01821243-x. [PubMed: 29434331].

3. Masoumi SZ, Poorolajal J, Keramat A, Moosavi SA. Prevalence of depression among infertile couples in Iran: a meta-analysis study. Iran J Public Health. 2013;42(5):458-66. [PubMed: 23802102].

4. Piwoński J, Piwońska A, Sygnowska E. Do depressive symptoms adversely affect the lifestyle? Results of the WOBASZ study. Kardiol Pol. 2010;68(8):912-8. [PubMed: 20730723].

5. Disu TR, Anne NJ, Griffiths MD, Mamun MA. Risk factors of geriatric depression among elderly Bangladeshi people: a pilot interview study. Asian J Psychiatr. 2019;44:163-9. doi: 10.1016/j.ajp.2019.07.050. [PubMed: 31382211].

6. Xie Y, Kong Y, Yang J, Chen F. Perfectionism, worry, rumination, and distress: a meta-analysis of the evidence for the perfectionism cognition theory. Personal Individ Differ. 2019:139:301-12. doi: 10.1016/j.paid.2018.11.028.

7. Kanchanatawan B, Thika S, Anderson G, Galecki P, Maes M. Affective symptoms in schizophrenia are strongly associated with neurocognitive deficits indicating disorders in executive functions, visual memory, attention and social cognition. Prog Neuropsychopharmacol Biol Psychiatry. 2018;80(Pt C):168-76. doi: 10.1016/j.pnpbp.2017.06.031. [PubMed: 28666826].

8. Haenisch B, Bönisch H. Depression and antidepressants: insights from knockout of dopamine, serotonin or noradrenaline re-uptake transporters. Pharmacol Ther. 2011;129(3):352-68. doi: 10.1016/j.pharmthera.2010.12.002. [PubMed: 21147164].

9. Richardson-Jones JW, Craige CP, Guiard BP, Stephen A, Metzger $\mathrm{KL}$, Kung HF, et al. 5-HT1A autoreceptor levels determine vulnerability to stress and response to antidepressants. Neuron. 2010;65(1):40-52. doi: 10.1016/j.neuron.2009.12.003. [PubMed: 20152112].

10. Gloster AT, Rinner MT, Ioannou M, Villanueva J, Block VJ, Ferrari G, et al. Treating treatment non-responders: a metaanalysis of randomized controlled psychotherapy trials. Clin Psychol Rev. 2020;75:101810. doi: 10.1016/j.cpr.2019.101810. [PubMed: 31884147].

11. McClintock SM, Brandon AR, Husain MM, Jarrett RB. A systematic review of the combined use of electroconvulsive therapy and psychotherapy for depression. J ECT. 2011;27(3):236-43. doi: 10.1097/YCT.0b013e3181faaeca. [PubMed: 21206376].

12. Kellner CH, Greenberg RM, Murrough JW, Bryson EO, Briggs MC, Pasculli RM. ECT in treatment-resistant depression. Am J Psychiatry. 2012;169(12):1238-44. doi: 10.1176/appi.ajp. 2012.12050648. [PubMed: 23212054].

13. Al-Harbi KS. Treatment-resistant depression: therapeutic trends, challenges, and future directions. Patient Prefer Adherence. 2012;6:369-88. doi: 10.2147/PPA.S29716. [PubMed: 22654508].

14. Strawbridge R, Arnone D, Danese A, Papadopoulos A, Vives AH, Cleare A. Inflammation and clinical response to treatment in depression: a meta-analysis. Eur Neuropsychopharmacol. 2015;25(10):1532-43. doi: 10.1016/j.euroneuro.2015.06.007. [PubMed: 26169573].

15. Croarkin PE, Wall CA, McClintock SM, Kozel FA, Husain MM, Sampson SM. The emerging role for rTMS in optimizing the treatment of adolescent depression. J ECT. 2010;26(4):323-9. doi: 10.1097/YCT.0b013e3181dd17eb. [PubMed: 20418774].

16. Hanley JR, Young AW. ELD revisited: a second look at a neuropsychological impairment of working memory affecting retention of visuo-spatial material. Cortex. 2019;112:172-9. doi: 10.1016/j.cortex.2018.10.029. [PubMed: 30527927].

17. Rahimi M. The effects of repetitive transcranial magnetic stimulation (rTMS) over the left DLPFC on neuropsychological function in women with depression. L'Encéphale. 2019;45:S689. doi: 10.1016/j.encep.2019.04.014.

18. Pirmoradi M, Dolatshahi B, Rostami R, Mohammadkhani $P$, Dadkhah A. Effect of repetitive transcranial magnetic stimulation (rTMS) in reducing severity of the signs and symptoms, such as cognitive, bodily and negativityworthlessness of depression in patients with recurrent major depression disorder. J Ardabil Univ Med Sci. 2013;13(2):174-86.

19. Durmaz O, Ebrinc S, Ates MA, Algul A. Evaluation of repetitive transcranial magnetic stimulation for treatment-resistant major depression and the impact of anxiety symptoms on outcome. Psychiatry Clin Psychopharmacol. 2017;27(1):14-8. doi: 10.1080/24750573.2017.1293239.

20. Mohabbat-Bahar S, Moradi-Joo M, Rayegani SM, Mashhadi A, Bigdeli I. Effectiveness of repetitive transcranial magnetic stimulation on working memory of patients with treatmentresistant major depression disorder. Res Med. 2017; 41(2):77-85.

21. Vanderhasselt MA, De Raedt R, Namur V, Lotufo PA, Bensenor IM, Boggio PS, et al. Transcranial electric stimulation and neurocognitive training in clinically depressed patients: a pilot study of the effects on rumination. Progr Neuropsychopharmacol Biol Psychiatry. 2015;57:93-9. doi: 10.1016/j.pnpbp.2014.09.015. [PubMed: 25455589].

22. Mahdavi A, Yazdanbakhsh K, Sharifi M. The effectiveness of mindfulness-based cognitive therapy in reducing psychological symptoms, meta-worry and thought fusion of multiple sclerosis patients. Annl Mil Health Sci Res. 2016;14(1):16-21.

23. Serafini G, Pompili M, Murri MB, Respino M, Ghio L, Girardi P, et al. The effects of repetitive transcranial magnetic stimulation on cognitive performance in treatment-resistant depression. A systematic review. Neuropsychobiology. 2015; 71(3):125-39. doi: 10.1159/000381351. [PubMed: 25925699].

24. Kaser M, Deakin JB, Michael A, Zapata C, Bansal R, Ryan D, et al. Modafinil improves episodic memory and working memory cognition in patients with remitted depression: a double-blind, randomized, placebo-controlled study. Biol Psychiatry Cogn Neurosci Neuroimaging. 2017;2(2):115-22. doi: 10.1016/ j.bpsc.2016.11.009. [PubMed: 28299368].

25. Cirillo P, Gold AK, Nardi AE, Ornelas AC, Nierenberg AA, Camprodon J, et al. Transcranial magnetic stimulation in anxiety and trauma-related disorders: a systematic review and meta-analysis. Brain Behav. 2019;9(6):e01284. doi: 10.1002/brb3.1284. [PubMed: 31066227].

26. Wu MI, Passos IC, Bauer IE, Lavagnino L, Cao B, Zunta-Soares $\mathrm{GB}$, et al. Individualized identification of euthymic bipolar disorder using the Cambridge Neuropsychological Test Automated Battery (CANTAB) and machine learning. J Affect Disord. 2016;192:219-25. doi: 10.1016/j.jad.2015.12.053. [PubMed: 26748737].

27. Asbaghi E, Rafieinia P, Hosseini SM, Sabahi P. The effectiveness of rTMS on working memory and symptoms of bipolar disorder. Neuropsychology. 2017;3(2):29-49.

28. TaheriFard M, Alizadeh GJ. The effectiveness of transcendental direct electric stimulation (TDCS) on improving cognitive functions and problem solving skills of students. J Sch Psychol. 2019;7(4):20-38.

29. Chen JJ, Zhao LB, Liu YY, Fan SH, Xie P. Comparative efficacy and acceptability of electroconvulsive therapy versus repetitive transcranial magnetic stimulation for major depression: a systematic review and multiple-treatments meta-analysis. Behav Brain Res. 2017;320:30-6. doi: 10.1016/j.bbr.2016.11.028. [PubMed: 27876667].

30. Kozel FA, Johnson KA, Nahas Z, Nakonezny PA, Morgan PS, Anderson BS, et al. Fractional anisotropy changes after several weeks of daily left high-frequency repetitive transcranial magnetic stimulation of the prefrontal cortex to treat major depression. J ECT. 2011;27(1):5-10. doi: 10.1097/YCT.0b013e 3181e6317d. [PubMed: 20559144].

31. Yang LL, Zhao D, Kong LL, Sun YQ, Wang ZY, Gao YY, et al. Highfrequency repetitive transcranial magnetic stimulation (rTMS) improves neurocognitive function in bipolar disorder. J Affect Disord. 2019;246:851-6. doi: 10.1016/j.jad.2018.12.102. [PubMed: 30795490]. 